\title{
Studentenkurs „Implantologie“ auf Burg Staufenberg
}

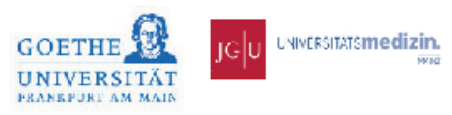

Keine Pflichtvorlesung auf harten Unibänken, sondern Kür in fürstlichem Umfeld: Die Universitäten Frankfurt und Mainz haben im März 2010 für besonders engagierte Studentinnen und Studenten einen außeruniversitären Implantologie-Kurs gestartet, in der Burg Staufenberg in Hessen dafür ein reizvolles Umfeld gefunden und mit dem Drei-Tage-Kompaktprogramm ein begehrtes Wissenspaket geschnürt: Der Startkurs in März war sofort ausgebucht, der Folgekurs Mitte September 2010 ebenfalls, nun geht das neue Projekt in Serie. Ab 2011 wird es zum festen Angebot.

Das Motto der Veranstaltungsreihe - „Fürstlich implantieren“ - spielt einerseits auf die Burg Staufenberg als Kursort an und signalisiert andererseits ein hohes Qualitätslevel in der implantologischen Ausbildung. Initiiert haben das Projekt Prof. Dr. mult. Robert Sader, Universität Frankfurt, und Prof. Dr. Dr. Bilal Al-Nawas, Universität Mainz. „Zusätzlich zur Universitätsausbildung sollen besonders interessierte Studentinnen und Studenten spezifisches Fachwissen erlangen und die Welt der Implantologie näher kennenlernen in einer inspirierenden Atmosphäre fernab vom Hörsaal", beschreibt Prof. Sader den Hintergrund der Veranstaltungsreihe. „Die speziell an der Implantologie interessierten Studentinnen und Studenten können sich in angenehmer Gruppengröße in lernintensiver Atmosphäre in die moderne Welt der Implantologie vertiefen." Realisiert wird das „fürstliche Implantieren“ in Zusammenarbeit mit CAMLOG, unterstützt wird das Projekt von den Unternehmen GEISTLICH und STOMA. An drei Tagen werden alle Facetten der Implantologie beleuchtet: Nach einer Einführung in die besondere Rolle von Ästhetik und Funktion folgen wissenschaftlich begründete aktuelle Positionen zu modernen Oberflächen, zu Implantat- und Abutmentgeometrien, Lösungen für Weichge- websprobleme und Vorgehensweisen bei knöchernen Augmentationen. Ergänzend zur Theorie bietet das Kurskonzept ausreichend Zeit für die Praxis: Am Schweinekiefer werden Schnitt-, Naht- und Lappentechniken geübt und am Kunststoffkiefer das Implantieren.

"Schwerpunkt ist die praxisnahe, wissenschaftlich untermauerte Planung implantatgetragener Rekonstruktionen sowohl aus chirurgischer als auch aus prothetischer Sicht," so Prof. Sader, "denn Interdisziplinarität spielt heute in der Implantatplanung eine entscheidende Rolle. Beide Seiten müssen berücksichtigt werden." Als besonders lehrreich empfinden die Studierenden das "Implantologie-Quiz", bei dem klinische Patientenfälle vorgestellt und dann verschiedene Therapieoptionen gemeinsam erarbeitet und ihre Vor- und Nachteile diskutiert werden. Eindrucksvoll für die Studenten sind dabei nicht zuletzt die freundschaftlichen, fachlich allerdings oft kontrovers geführten Diskussionen, die auf unterschiedliche Konzepte der beiden veranstaltenden Universitätskliniken zurückgehen.

Infos und Anmeldungen für den nächsten Kurs vom 17. - 19. März 2011 unter

入 Tel. 07044-9445667 nicole.guenthner@camlog.com www.camlog.com

\section{Pharmatechnik}

\section{Software: moderner - schöner - einfacher}

Der Servicegedanke steht bei LinuDent an erster Stelle. Daher wurde die innovative Software - LinuDent - für die Kunden moderner und zukunftssicherer gestaltet. Das neue LinuDent tritt nicht nur in einem neuen Gewand auf, auch die Bedienerfreundlichkeit wurde weiter optimiert. Die zahnmedizinische Verwaltungsassistentin Sabine Lichtner aus der Praxis Dr. Isa Rait in München hat die neue Ausführung bereits getestet: „Diese Version überzeugt durch noch mehr Benutzerfreundlichkeit und ist klarer und strukturierter als die vorherige Version. Besonders positiv empfinde ich die „Augenfreundlichkeit" der neuen

\section{LinuDent}

powered by PHARMATECHNIK

Generation. Die einzelnen Symbole sind nun in der Größe veränderbar und können je nach Belieben angepasst werden. Generell ist die gesamte Oberfläche individueller zu gestalten. Alles in allem würde ich sagen, dass viele kleine Details geändert wurden, die das Leben deutlich erleichtern."

ス Tel. 08151 - 4442-564

info@linudent.de www.linudent.de

\section{Humanchemie}

\section{Gewinnspiel zum Firmenjubiläum}

Am 06.11.2010 feiert die familiengeführte Humanchemie $\mathrm{GmbH}$ bereits das 35-jährige Firmenjubiläum. Das vor allem durch die Präparate Tiefenfluorid ${ }^{\oplus}$ und Cupra $^{\circledast}$ bzw. Depotphorese ${ }^{\circledast}$ mit Cupra $^{\circledast}$ bekannte Unternehmen wird dieses Jubiläum mit einem großen Gewinnspiel begehen. Nach dem Motto 35 Jahre - 35 Preise winken z. B. eine Woche im 4-Sterne-Hotel an der Ostsee oder ein Wellness-Wochenende für zwei Personen.

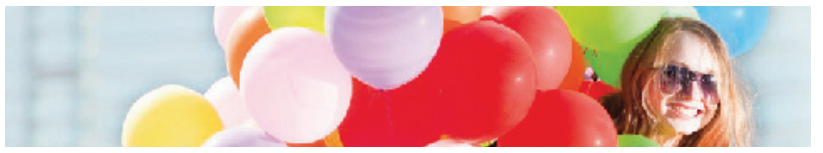

Außerdem sucht das Team der Humanchemie GmbH das älteste Depotphorese ${ }^{\circledR}$-Gerät. Der Besitzer darf mit einer Überraschung rechnen.

Weitere Informationen erhalten Sie an den Messeständen der Herbstmessen, sowie direkt bei der Humanchemie GmbH.

入 Tel. 05181- 24633

info@humanchemie.de

www.humanchemie.de 